\title{
Produções científicas sobre as avaliações no âmbito da Atenção Primária à Saúde no Brasil: uma revisão sistemática
}

\author{
Scientific productions on the evaluation in the primary health care in Brazil: a systematic review

\section{Producciones cientificas sobre la evaluación de la atención primaria de salud en Brasil: una revisión sistemática}

Ane Polline Lacerda Protasio. Universidade Federal da Paraíba (UFPB). João Pessoa, PB, Brasil. anepolline@hotmail.com (Autora correspondente). Liliane dos Santos Machado. Universidade Federal da Paraíba (UFPB). João Pessoa, PB, Brasil. liliane@di.ufpb.br

Ana Maria Gondim Valença. Universidade Federal da Paraíba (UFPB). João Pessoa, PB, Brasil. anamvalenca@gmail.com

\section{Resumo}

Objetivo: neste momento em que se discute a reorganização dos serviços de saúde do Brasil, o tema da avaliação da atenção primária, em seus termos mais amplos, ganha relevância. Para contribuir para a área, este trabalho descreve uma revisão sistemática que objetivou caracterizar e analisar os processos avaliativos da atenção primária no Brasil. Métodos: a busca de artigos foi feita nas bases de dados PubMed, Lilacs e Scielo, a partir dos descritores "Primary Health Care AND Health Evaluation AND Brazil" restringido o período aos últimos cinco anos (2009 a 2013). Resultados: com a configuração de pesquisa, foram incluídos 42 artigos cujo cenário era a atenção primária no Brasil e que, em suas análises, faziam referência à avaliação dos serviços e programas. Observou-se uma diversidade de metodologias e objetivos dessas avaliações, destacando-se a avaliação dos serviços fornecidos pela Estratégia Saúde da Família (38,1\%), a avaliação de serviços (61,9\%), o foco no usuário (9,5\%) e as pesquisas quantitativas (76\%). Os estudos foram realizados predominantemente nas regiões Nordeste $(n=15)$ e Sudeste $(n=13)$. Conclusão: em função dos achados, pode-se afirmar que as avaliações na atenção primária no Brasil ainda são restritas e localizadas, merecendo ser ampliadas e efetivamente aplicadas, para, assim, aperfeiçoar-se continuamente este processo.

\section{Abstract}

Objective: nowadays, when the reorganization of health services in Brazil is being discussed, the theme of Primary Health Care Evaluation, in its broadest terms, becomes relevant. In order to contribute to this area, this paper describes a systematic review that characterizes and analyzes the evaluation processes of Primary Health Care in Brazil. Methods: we searched PubMed, Lilacs and SciELO databases using the descriptors "Primary Health Care AND Health Evaluation AND Brazil", restricted to the period of the last five years (2009-2013). Results: with this configuration, we identified 42 articles about Primary Health Care in Brazil that focused on the evaluation of services and programs. We noted a variety of methodologies and objectives: $38.1 \%$ focused on the evaluation of health programs in Brazil's Family Health Strategy, $61.9 \%$ on the evaluation of health services, $9.5 \%$ on the users' point of view and $76 \%$ in quantitative surveys. The studies were conducted primarily in the Northeast $(n=15)$ and Southeast $(n=13)$. Conclusion: the evaluations of the Brazil's Primary Health Care are still very limited and localized. They deserve to be expanded and effectively applied continuously to increase the results obtained.

\section{Resumen}

Objetivo: en este momento en que se analiza la reorganización de los servicios de salud en Brasil, el tema de la evaluación de la Atención Primaria, en sus términos más amplios, cobra mayor relevancia. Con el fin de contribuir a la área de conocimiento, en el presente trabajo se describe un estudio de la literatura, por medio de una revisión sistemático, con el objetivo de caracterizar y analizar los procesos de evaluación de la atención primaria de salud en Brasil. Métodos: la búsqueda de los artículos en PubMed, Lilacs y Scielo, de los descriptores "Atención primaria de la Salud y Evaluación de la Salud y Brasil” restringido el período de los últimos cinco años (2009 a 2013). Resultados: con esta configuración de búsqueda, se incluyeron 42 artículos cuyo escenario fue la atención primaria de Salud en Brasil y que, en su análisis, hizo referencia a la evaluación de los servicios y programas. Se observó que había una diversidad de metodologías y objetivos de estas evaluaciones con evidencia en los programas realizados en la Estrategia de Salud Familiar (38,1\%), en la evaluación de los servicios $(61,9 \%)$, en el enfoque en el usuario $(9,5 \%)$ y en los estudios de tipo cuantitativo (76\%). La ubicación mayoritaria fue en las regiones Noreste $(n=15)$ y Sureste $(n=13)$. Conclusión: las evaluaciones de la Atención Primaria de Salud del Brasil siguen siendo muy limitadas y localizadas, merecen ser ampliadas y efectivamente aplicadas de una forma continua para incrementar los resultados obtenidos.

Como citar: Protásio APL, Machado LS, Valença AMG. Produções científicas sobre as avaliações
Fonte de financiamento: declaram não haver. Parecer CEP: não se aplica.

Conflito de interesses: declaram não haver. Recebido em: 21/12/2014. Aprovado em: 16/09/2014. 


\section{Introdução}

A Conferência Mundial de Saúde em Alma-Ata, realizada em 1978, constituiu-se em um marco na saúde pública mundial, pois estabeleceu o consenso de que a Atenção Primária à Saúde (APS) seria a estratégia fundamental e a porta de entrada dos sistemas de saúde. ${ }^{1}$ Desde então, vem ocorrendo, em todo o mundo, o fortalecimento da APS como eixo orientador de organização dos sistemas de saúde e otimização dos recursos disponíveis. ${ }^{2-5}$

No Brasil, na década de 1990, com a institucionalização da Constituição de 1988, o processo de descentralização da saúde e o destaque assumido pela APS na agenda setorial promoveram importantes mudanças na política nacional de atenção primária à saúde, sendo evidenciado um expressivo investimento na ampliação do acesso à saúde. Pode-se dizer que tais transformaçôes ganharam força com a aprovação da Norma Operacional Básica do SUS de 1996 (NOB-SUS 01/96), do Programa Agentes Comunitários de Saúde (PACS) e do Programa Saúde da Família (PSF), estratégias prioritárias para o fortalecimento da atenção primária nos sistemas municipais de saúde. ${ }^{6}$

De acordo com o Ministério da Saúde, ${ }^{7}$ a atenção primária (também denominada atenção básica) constitui-se em um conjunto de açôes de saúde que englobam a promoção, prevenção, diagnóstico e prestação de cuidado (tratamento e reabilitação), desenvolvidas por intermédio do exercício de práticas gerenciais, democráticas, participativas e sanitárias. A atenção primária deve ter uma abordagem transdisciplinar, dirigida a populaçóes de territórios bem delimitados, sob a forma de trabalho em equipe, a qual assume responsabilidades que, utilizando tecnologias de elevada complexidade e baixa densidade, devem resolver a maioria dos problemas de saúde das populaçôes (de maior frequência e relevância). Esta atençáo primária deve ser orientada pelos princípios da universalidade, acessibilidade, continuidade, integralidade, responsabilização, humanização, vínculo, equidade e participação social. No cenário de fortalecimento da atenção primária, torna-se evidente a necessidade e o interesse em avaliar e monitorar os resultados alcançados em relação à organização e à provisão dos serviços, bem como os possíveis impactos produzidos na saúde e no bem-estar das populaçóes. ${ }^{8}$

A avaliaçáo em saúde apresenta uma pluralidade de conceitos e práticas por se constituir como um processo críticoreflexivo, contínuo e sistemático desenvolvido no âmbito dos serviços de saúde. Pelo fato de a avaliação em saúde não ser um procedimento exclusivamente de natureza técnica, embora essa dimensão esteja presente, é preciso que a mesma tenha como prática o desenvolvimento de negociação entre atores sociais. Nesse sentido, ela deve fazer parte do conjunto de atividades desempenhadas pelos gestores do sistema e das equipes de saúde. Ademais, é importante que a avaliação possibilite a percepção de mudanças na perspectiva de reorganização do sistema e reorientação das práticas de saúde. ${ }^{7}$

Entretanto, os estudos que avaliam a atenção primária no Brasil são poucos, e os existentes priorizam causas específicas e focadas na heterogeneidade das situaçóes a serem avaliadas. Apesar dessas iniciativas de caráter mais abrangente, a experiência na área de monitoramento e avaliação no SUS ainda é incipiente. ${ }^{?}$

Neste momento em que se discute a reorganização dos serviços de saúde do Brasil, o tema da avaliação da atenção primária, em seus termos mais amplos, ganha relevância. Para contribuir para a área, este trabalho descreve uma revisão sistemática da literatura com o objetivo de caracterizar e analisar os processos avaliativos da atençâo primária no Brasil.

\section{Métodos}

O presente estudo é uma pesquisa sistemática de artigos publicados na literatura científica tendo como elemento-chave a seguinte questão: como as pesquisas que avaliam a atenção primária à saúde no Brasil são estruturadas?

A fim de identificar e de analisar as pesquisas avaliativas sobre a atenção primária à saúde no Brasil, foram pesquisadas as bases de dados MEDLINE (Medical Literature Analysis and Retrieval System Online) via Pubmed, Scielo (Scientific Eletronic Library Online) e Lilacs (Literatura Latino-Americana e do Caribe em Ciências da Saúde), devido ao impacto de seus artigos.

Foram escolhidos os seguintes descritores, a partir do site DeCS (Descritores em Ciências da Saúde): "Primary Health Care AND Health Evaluation AND Brazil" utilizados no campo "índice de assuntos”. Adicionalmente, foi restringido o período de pesquisa aos últimos cinco anos (2009 a setembro de 2013), sendo entáo selecionados artigos indexados com textos disponíveis e pesquisas em humanos.

$\mathrm{Na}$ Figura 1 é vista a estrutura da configuração da pesquisa. 


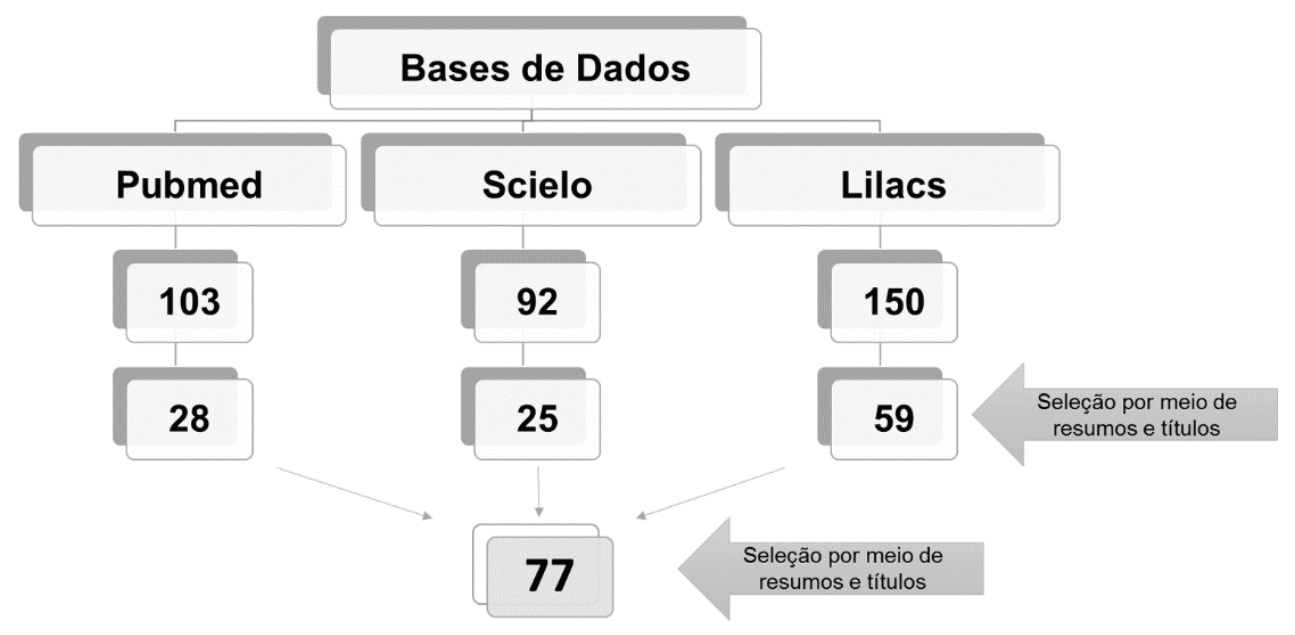

Figura 1. Estrutura da configuração da pesquisa.

Como visto na Figura 1, obteve-se resposta de 103 artigos na Pubmed, 92 artigos na Scielo e 150 artigos no Lilacs, atingindo-se o total de 345 artigos. Em seguida, realizou-se a leitura dos títulos e dos resumos desses artigos e aplicaram-se os seguintes critérios de elegibilidade:

- foram excluídos artigos que não envolviam avaliaçóes ou que não avaliavam os serviços de saúde da atenção primária no Brasil;

- foram excluídos os artigos que se referiam a diagnóstico, determinantes, terapêutica, protocolo de atendimento, modelo de intervenção, relato de experiência, vigilância, monitoramento ou histórico;

- foram excluídos os artigos que se repetiam nessas bases.

A aplicação destes critérios resultou em 77 artigos indexados. Esses artigos foram lidos integralmente, sendo que, após uma análise metodológica, foram incluídos apenas aqueles que continham pesquisas cujo cenário era a atençáo primária à saúde no Brasil e que, em suas análises, faziam referência à avaliação dos serviços e programas.

Após a considerção de todos os critérios, amostra final constou de 42 publicaçôes, que foram sistematicamente revisadas em relação à sua adequação ao tema. Posteriormente, os estudos foram apresentados de forma descritiva, com base em classificação, segundo categorias propostas, por meio de frequências absolutas e relativas.

No planejamento desta revisão sistemática, foram estipuladas e organizadas categorias analíticas para compilação dos dados. $\mathrm{O}$ resultado dessa sistematização gerou sete categorias:

I) ano de publicação;

II) local onde o estudo foi desenvolvido;

III) foco da avaliação (referente ao objetivo da avaliaçáa);

IV) periódico;

V) tipo de estudo (instrumento de coleta quantitativo, qualitativo ou misto);

VI) amostra (dados, gestores, usuários, profissionais de saúde); e

VII) tipo de avaliação (avaliação de programas ou serviços).

\section{Resultados}

No Quadro 1, pode ser visualizada a caracterização das produçóes científicas publicadas no intervalo entre 2009 e 2013, segundo autor/ano, foco da avaliaçáo, tipo de estudo e instrumento de coleta. 
Quadro 1. Caracterização das produções científicas.

\begin{tabular}{|c|c|c|c|}
\hline Autor/Ano & Foco da Avaliação & Tipo de Estudo & Instrumento de Coleta \\
\hline Silva et al. $(2013)^{10}$ & Cuidado pré-natal & Transversal e quantitativo & Entrevista Estruturada \\
\hline Sousa et al. (2012) $)^{11}$ & Atenção Farmacêutica na APS & Transversal e quantitativo & Entrevista Estruturada \\
\hline Santos Neto et al. (2012) ${ }^{12}$ & $\begin{array}{l}\text { Práticas integrativas e complementares } \\
\text { no SUS }\end{array}$ & Estudos de Caso quantitativo & Dados e Questionário Semi-estruturado \\
\hline Raposo; Nemes (2012) ${ }^{13}$ & $\begin{array}{l}\text { Assistência pré-natal nos cartões de } \\
\text { gestantes }\end{array}$ & Transversal e quantitativo & Entrevista \\
\hline Júnior; Nunes (2012) ${ }^{16}$ & Atenção primária a menores de 5 anos & Transversal e quantitativo & $\begin{array}{l}\text { Entrevista } \\
\text { Semi-estruturada }\end{array}$ \\
\hline Costa; Pereira (2012) $)^{17}$ & $\begin{array}{l}\text { Assistência farmacêutica na atenção } \\
\text { primária }\end{array}$ & Longitudinal, quantitativo e qualitativo & Entrevista Estruturada \\
\hline Rabetti; Freitas $(2011)^{20}$ & Hipertensão na ESF & Transversal e quantitativo & Dados \\
\hline Pereira et al. (2011) $)^{21}$ & $\begin{array}{l}\text { Organizacionais e desempenho, da rede } \\
\text { da ABS }\end{array}$ & Transversal e quantitativo & Questionário Validado \\
\hline Mendoza-Sassi et al. $(2011)^{22}$ & Pré-natal na ESF e nas UBS tradicionais & Transversal e quantitativo & Entrevista Estruturada \\
\hline Leão et al. $(2011)^{23}$ & Atenção infantil na APS & Transversal e quantitativo & Questionário Validado \\
\hline Lafaiete et al. $(2011)^{24}$ & $\begin{array}{l}\text { Programa de Controle da Tuberculose na } \\
\text { UBS }\end{array}$ & Transversal e quantitativo, & Questionário Validado \\
\hline Gomes et al. $(2011)^{25}$ & $\begin{array}{l}\text { Ações e serviços de saúde oferecidos pela } \\
\text { ESF }\end{array}$ & Transversal, quantitativo e qualitativo & Entrevista \\
\hline Costa et al. $(2011)^{26}$ & Hipertensão arterial na ESF & $\begin{array}{l}\text { Estudos de avaliação normativa e } \\
\text { Quantitativo }\end{array}$ & Dados e Entrevista Estruturada \\
\hline Serra; Rodrigues (2010) $)^{31}$ & Referência e contra-referência na ESF & Transversal, quantitativo e qualitativo & Dados e Entrevista Semi-estruturada \\
\hline Mendonça et al. $(2010)^{32}$ & Gestão do trabalho na USF & Estudos de Caso, qualitativo e quantitativo & Entrevista Semi-estruturada \\
\hline Lima et al. $(2010)^{33}$ & Dados antropométricos produzidos na APS & Transversal e quantitativo & Medidas Antropométricas \\
\hline Lana; Lima $(2010)^{34}$ & Pré-natal e transmissão vertical do nas UBS & Transversal e quantitativo & Dados \\
\hline Hoepfner; Franco $(2010)^{35}$ & Hipertensão arterial nas UBS & Transversal e quantitativo & Dados \\
\hline Fischer et al. (2010) ${ }^{36}$ & Indicadores de saúde bucal no SUS & Ecológico e quantitativo & Dados \\
\hline Cunha; Vieira-da-Silva (2010) ${ }^{37}$ & Acessibilidade à atenção primária & Estudo de Caso quantitativo & Entrevista Semi-estruturada \\
\hline Amaral et al. $(2010)^{38}$ & $\begin{array}{l}\text { Programa Nacional de Controle da } \\
\text { Tuberculose }\end{array}$ & Avaliação normativa e quantitativa & Dados \\
\hline Tavares et al. $(2009)^{39}$ & Expansão da ESF & Transversal, e qualitativo & Grupos Focais e Observação \\
\hline Scatena et al. $(2009)^{40}$ & $\begin{array}{l}\text { Acesso ao diagnóstico da tuberculose na } \\
\text { APS }\end{array}$ & Transversal e quantitativo & Questionário Validado \\
\hline Pereira et al. (2009) ${ }^{41}$ & Equipe de saúde bucal na ESF & Longitudinal e quantitativo & Entrevista Estruturada \\
\hline Nascimento et al. (2009) ${ }^{42}$ & Saúde bucal após a ESF. & Transversal e qualitativo & Grupos Focais \\
\hline Nagahama $(2009)^{43}$ & Saúde reprodutiva na APS. & Transversal e quantitativo & Questionário Transversal \\
\hline Motta et al. $(2009)^{44}$ & $\begin{array}{l}\text { Acesso ao diagnóstico da tuberculose na } \\
\text { APS }\end{array}$ & Transversal e quantitativo & Entrevista \\
\hline Medina; Hartz (2009) ${ }^{45}$ & Implantação da APS na ESF & Transversal e quantitativo & Dados e Entrevista Semi-estruturada \\
\hline Gouveia et al. $(2009)^{46}$ & Satisfação dos usuários no SUS & Transversal e quantitativo & Dados \\
\hline
\end{tabular}


Quadro 1. Continuação...

\begin{tabular}{|c|c|c|c|}
\hline Autor/Ano & Foco da Avaliação & Tipo de Estudo & Instrumento de Coleta \\
\hline Gomes et al. $(2009)^{47}$ & 0 impacto da ESF & Transversal e quantitativo & Entrevista Estruturada \\
\hline Finkelsztejn et al. (2009) ${ }^{48}$ & $\begin{array}{l}\text { Encaminhamentos realizados pela rede de } \\
\text { APS }\end{array}$ & Transversal e quantitativo & Dados \\
\hline Figueiredo et al. (2009) ${ }^{49}$ & Controle da tuberculose na APS & Transversal e quantitativo & Questionário Validado \\
\hline Fernandes et al. (2009) $)^{50}$ & Internações sensíveis ao cuidado da ESF & Transversal e quantitativo & Dados e Entrevista \\
\hline Almeida; Tanaka (2009) $)^{51}$ & $\begin{array}{l}\text { Programa de Humanização do Pré-Natal e } \\
\text { Nascimento }\end{array}$ & Transversal, quantitativo e qualitativo & Grupos Focais e Dados \\
\hline
\end{tabular}

Nos 42 artigos sobre avaliação da atenção primária à Saúde no Brasil, observou-se uma diversidade de objetivos dessas avaliações, destacando-se a avaliação dos serviços fornecidos pela Estratégia Saúde da Família. Além disso, as pesquisas contemplaram avaliações de atenção à saúde, implantação, desempenho, completude de informaçôes, oferta e produção, satisfação, eficiência, organização, acessibilidade, gestão técnica e processos de trabalho.

Quanto ao foco das avaliaçóes, verificou-se que 38,1\% das pesquisas avaliaram programas, principalmente os referentes à Estratégia Saúde da Família, assim como aqueles relacionados ao Programa Nacional de Controle da Tuberculose, Programa de Humanizaçáo do Pré-Natal e Nascimento, Programa de Hanseníase e Programa de Controle da Asma. O restante, 61,9\%, avaliou os serviços ofertados, conforme descrito na Tabela 1.

Tabela 1. Distribuição dos artigos segundo a Região Geográfica e Amostra.

\begin{tabular}{llcc}
\hline \multirow{2}{*}{ Gegião } & Centro-oeste & $\begin{array}{c}\text { Frequência } \\
\text { Absoluta (n) }\end{array}$ & $\begin{array}{c}\text { Frequência } \\
\text { Relativa (\%) }\end{array}$ \\
\hline \multirow{7}{*}{ Amostra } & 1 & 2,4 \\
& Nordeste & 15 & 35,7 \\
& Sudeste & 13 & 31,0 \\
& Sul & 9 & 21,4 \\
& Mais de 2 regiões & 4 & 9,5 \\
& Total & 42 & 100,0 \\
& Gados & 7 & 16,7 \\
& Usuários & 2 & 4,8 \\
& Profissionais de saúde & 16 & 38,1 \\
& Profissionais de saúde e gestores & 7 & 16,7 \\
& Profissionais de saúde e dados & 2 & 4,8 \\
& Profissionais de saúde e usuários & 1 & 2,4 \\
& Usuários e dados & 1 & 9,5 \\
& Profissionais de saúde, usuários e dados & 2 & 2,4 \\
& Total & 42 & 4,8 \\
& & & 100,0 \\
\hline
\end{tabular}

Os periódicos "Cadernos de Saúde Pública", "Revista de Saúde Pública" e "Ciência \& Saúde Coletiva” foram aqueles em que se localizou o maior número de artigos nesses estudos que avaliaram a atenção primária, seguidos por outros, também de saúde pública, os quais compunham 75,6\% dos periódicos analisados nesta revisão sistemática.

Nos artigos selecionados, encontraram-se diferentes desenhos metodológicos para avaliar a atenção primária, destacando-se estudos transversais e coletas de informação por meio de dados ou entrevistas ou da união entre estes. Pesquisas quantitativas foram as mais frequentes $(76 \%)$ e, em sua maioria, utilizaram entrevistas semiestruturadas ou estruturadas. Nos estudos qualitativos, encontrou-se a presença de grupos focais como alvo de consulta. Sete estudos utilizaram método quantitativo e qualitativo. Apenas quatro pesquisas foram de estudos de caso e duas de estudos ecológicos.

$\mathrm{Na}$ Tabela 1, encontra-se a distribuição dos artigos segundo a regiáo geográfica e amostra. Entre os artigos pesquisados, não foi observada uma cidade de destaque em número de avaliaçôes. Porém, as regióes que apresentaram o maior número de estudos publicados foram a Nordeste $(n=15)$ e a Sudeste $(n=13)$. Os resultados das outras localizaçóes foram: região Sul com $n=9$, região Centro-Oeste com $n=1$ e o estado do Rio Grande do Sul com $n=1$. Quatro artigos abrangeram mais de uma regiáo, sendo que, entre os mesmos, apenas um avaliou cidades das cinco regióes do país. 
Neste estudo, encontraram-se diferentes alvos de consulta das informaçóes, sendo o principal deles os usuários $(38,1 \%)$, seguido por dados secundários (16,7\%), profissionais de saúde (16,7\%) e gestores (4,8\%). Algumas pesquisas utilizaram a combinaçôes destes alvos de consulta, a saber: profissionais de saúde e usuários $(9,5 \%)$, profissionais de saúde e gestores $(4,8 \%)$, profissionais de saúde e dados $(2,4 \%)$, usuários e dados $(2,4 \%)$, e profissionais de saúde, usuários e dados $(2,4 \%)$.

Nos estudos com dados secundários (17\%), foram encontrados, principalmente, os sistemas de informação do Ministério da Saúde, tais como CNES (Cadastro Nacional de Estabelecimentos de Saúde), SIA (Sistema de Informaçóes Ambulatoriais), SINAN (Sistema de Informação de Agravos de Notificação), SIAB (Sistema de Informação da Atenção Básica), DATASUS (Banco de dados do Sistema Único de Saúde), SINASC (Sistema de Informações sobre Nascidos Vivos), SIM (Sistema de Informaçóes sobre Mortalidade) e SISPRENATAL (Sistema de Acompanhamento do Programa de Humanização no Pré-Natal e Nascimento).

\section{Discussão}

A avaliação em saúde pode desdobrar-se em diferentes concepçóes sobre saúde e práticas de saúde, as quais, à semelhança de outras práticas sociais, podem constituir-se de objeto de avaliação nas suas diversas dimensôes, devendo ocorrer desde o cuidado individual até os estados mais complexos de intervençáo e de organizaçáo, como políticas, programas, serviços ou sistemas. ${ }^{52}$ Ao se analisar o conjunto de pesquisas avaliativas, foi possível confirmar essa diversidade no foco das pesquisas no Brasil.

A Estratégia Saúde da Família foi o principal tema da maioria das pesquisas, apesar de ser tratada de diferentes maneiras, sendo seu foco a implantação da própria estratégia ou programas e projetos desenvolvidos nela. Verificou-se que importantes linhas de cuidado foram avaliadas, tais como: saúde bucal, saúde da mulher, saúde mental, saúde da criança, hipertensão, tuberculose e pré-natal, o que revela a preocupação com os fluxos assistenciais seguros e garantidos ao usuário, no sentido de atender às suas necessidades de saúde. No entanto, a maioria dos trabalhos envolve apenas unidades de saúde em número limitado, o que faz com que essas avaliaçóes náo reflitam a real situaçáo da populaçáo em geral.

As avaliaçôes envolvendo pré-natal foram igualmente distribuídas durante os últimos cinco anos na literatura ${ }^{10,13,22,34,51}$, período durante o qual foram analisados o desempenho e a qualidade desse serviço a fim de garantir a efetividade no cuidado à gestante e reduzir resultados obstétricos indesejáveis. Dois estudos avaliaram a qualidade do pré-natal com base na tríade de Donabedian ${ }^{53}$ "estrutura-processo-resultados" para conceituar a qualidade da atenção. Essa tríade, muito usada em avaliaçóes de saúde, foi criada por Donabedian com o intuito de aferir a qualidade do cuidado médico, e foi assim explicada: a "estrutura" corresponderia às características relativamente estáveis dos seus provedores, aos instrumentos e recursos, bem como às condiçôes físicas e organizacionais; o "processo" corresponderia ao conjunto de atividades desenvolvidas na relação entre profissionais e pacientes; e os "resultados" seriam as mudanças verificadas no estado de saúde dos pacientes que pudessem ser atribuídas a um cuidado prévio.

Dois estudos publicados em 2012 avaliaram o serviço de atenção farmacêutica da atençáo básica, deixando evidente a importância de um profissional que se responsabilize pela farmacoterapia dos pacientes de modo a propiciar maior segurança para o processo, além da necessidade de um gerenciamento efetivo para evitar desperdícios de recursos para os municípios. Ambos os estudos foram qualitativos e observaram os achados sob a ótica dos profissionais de saúde da atenção primária. ${ }^{11,17}$

Apenas um dos trabalhos pesquisados avaliou as características da oferta e produção de práticas integrativas e complementares no SUS, destacando que a implantação dessas práticas na atenção primária ainda encontra dificuldades devido à insuficiente produção e pesquisa, às limitaçôes no controle dessas práticas, à pouca formação e à carência de especialistas na área ${ }^{6}$. Isso explicaria, então, a escassez de estudos avaliativos dessa área nos últimos cinco anos no âmbito da atenção primária à saúde.

Entre as pesquisas com dados primários, observou-se que a consideração de diversos atores privilegiados, sendo o principal deles os usuários, é uma forte tendência na avaliação de programas de saúde pública executados exclusivamente por especialistas; entre as avaliaçôes, observou-se uma que considerou os beneficiários como atores-chave dos processos. ${ }^{54}$ Para Serapione, ${ }^{55}$ a visáo do usuário constitui-se em um elemento central no processo avaliativo, haja visto que o produto dos serviços de saúde resulta da interação entre usuários, profissionais de saúde, gestores e todo o contexto que envolve as relaçôes entre eles. 
De 2007 a 2011, verificaram-se 5 trabalhos publicados que avaliaram o atendimento ao portador de tuberculose quanto ao acesso, ao tratamento e ao controle da tuberculose. Todos esses trabalhos consistiram em pesquisas quantitativas, e $80 \%$ deles tiveram como foco a percepçáo dos usuários.

Embora a maioria das pesquisa encontradas fossem do tipo quantitativo, verificou-se no presente estudo que 7 pesquisas utilizaram método qualitativo e quantitativo. Esse fato mostrou-se bastante interessante, pois essa dupla abordagem no âmbito da avaliação em saúde, segundo a qual os métodos diversos não são considerados antagônicos ou "alternativos", mas distinguem-se ontologicamente e se complementam: no método quantitativo, o fenômeno avaliado revela os aspectos objetivos, enquanto que no qualitativo, é aprofundada a compreensão destes fenômenos. ${ }^{56}$

Os artigos encontrados no presente estudo corroboram com Santos-Filho, ${ }^{57}$ que observou tendências e inovaçóes no campo da avaliação em saúde para facilitar a tomada de decisão e a gestão. Há um destaque maior para mudança na atenção primária a qual está em processo de reestruturação com introdução de novas estratégias, entre essas, a avaliação como prática diária, evidenciando a disponibilidade da atenção, o acesso aos serviços e a resolutividade, numa perspectiva integral da saúde. Vitoria et a ${ }^{8}$ sugerem que a avaliação da APS no Brasil incorpore a análise de adequaçáo da estrutura e do processo, pois a obtenção dos recursos financeiros necessários, assim como ambientes agradáveis, tempos de espera toleráveis e superação das carências estruturais, são fatores essenciais para se transformar a APS na real porta de entrada do SUS.

\section{Conclusão}

Observa-se que há uma dificuldade de conduzir e implementar processos de avaliação na atenção primária uma vez que existe uma diferença entre a concepção da atenção primária e o que está sendo exercido na prática dos serviços de saúde. A produção de um conhecimento, na pesquisa de avaliação deve ter como componente indispensável o processo de planejamento e, nesse sentido, não é meramente atribuição de avaliadores externos, devendo fazer parte do conjunto de atividades desempenhadas pelos gestores do sistema e profissionais envolvidos. ${ }^{59}$ Entretanto, com o presente estudo foi possível concluir que as avaliaçóes na atençáo primária no Brasil ainda são restritas e localizadas, merecendo ser ampliadas, efetivamente aplicadas, e aperfeiçoadas continuamente em função dos resultados encontrados.

\section{Referências}

1. WHO. Declaration of Alma-Ata, 1978 [acesso em 2013 Aug 03]. Geneva: World Health Organization; 1978. Disponível em: http://www.paho.org/hq/index.php?option=com_docman\&task=doc_view\&gid=19004

2. Rivero DAT. Alma-Ata: 25 años después. Perspect Salud. 2003;8:2-7.

3. Bursztyn I, Kushnir R, Giovanella L, Stolkiner A, Sterman-Heimann L, Riveros Ml, et al. Notas para el estudio de la Atención Primaria en contextos de sistemas de salud segmentados. Rev Salud Pública Bogotá. 2010;12:77-88. http://dx.doi.org/10.1590/S0124-00642010000700006

4. Pereira AMM, Castro ALB de, Malagón Oviedo RA, Barbosa LG, Gerassi CD, Giovanella L. Primary health care in South America in comparative perspective: changes and trends. Saúde Debate. 2012;36:482-99. http://dx.doi.org/10.1590/S0103-11042012000300019

5. WHO. The World Health Report 2008 - primary Health Care (Now More Than Ever) [Internet]. WHO; 2008. Disponível em: http://www.who.int/whr/2008/en/index.html

6. Castro ALB, Machado CV. The federal primary health care politics in Brazil in the 2000's. Physis Rev Saúde Coletiva. 2012;22:477-506. http://dx.doi.org/10.1590/S0103-73312012000200005

7. Ministério da Saúde (BR), Coordenação de Acompanhamento e Avaliação, Departamento de Atenção Básica, Secretaria de Atenção à Saúde. Documento final da Comissão de Avaliação da Atenção Básica. Brasília: MS; 2003. Disponível em: http://bvsms.saude.gov.br/bvs/publicacoes/avaliacao_ab_portugues.pdf

8. Almeida PF, Giovanella L. Avaliação em Atenção Básica à Saúde no Brasil: mapeamento e análise das pesquisas realizadas e/ou financiadas pelo Ministério da Saúde entre os anos de 2000 e 2006. Cad Saúde Pública. 2008;24(8):1727-42. http://dx.doi.org/10.1590/S0102-311X2008000800002

9. Santos-Filho SB. Perspectives of the evaluation of Brazil's National Health Humanization Policy: conceptual and methodological aspects. Ciênc Saúde Coletiva. 2007;12(4):999-1010. http://dx.doi.org/10.1590/S1413-81232007000400021

10. Silva EP, Lima RT, Costa MJC, Batista Filho M. Development and application of a new index for assessment of prenatal care. Rev Panamericana Salud Pública. 2013;33(5):356-62. http://dx.doi.org/10.1590/S1020-49892013000500007 
11. Sousa IMC, Bodstein RCA, Tesser CD, Santos FAS, Hortale VA. Integrative and complementary health practices: the supply and production of care in the Unified National Health System and in selected municipalities in Brazil. Cad Saúde Pública. 2012;28(11):2143-54. http://dx.doi.org/10.1590/S0102-311X2012001100014

12. Santos Neto ET, Oliveira AE, Zandonade E, Gama SGN, Leal MC. [Prenatal patient cards and quality of prenatal care in public health services in Greater Metropolitan Vitória, Espírito Santo State, Brazil]. Cad Saúde Pública. 2012;28(9):1650-62. http://dx.doi.org/10.1590/S0102$311 \times 2012000900005$

13. Raposo MT, Nemes MIB. Assessment of integration of the leprosy program into primary health care in Aracaju, state of Sergipe, Brazil. Rev Soc Bras Med Tropical. 2012;45(2):203-8. http://dx.doi.org/10.1590/S0037-86822012000200013

14. Onocko-Campos RT, Campos GWS, Ferrer AL, Corrêa CRS, Madureira PR, Gama CAP, et al. Evaluation of innovative strategies in the organization of Primary Health Care. Rev Saúde Pública. 2012;46(1):43-50. http://dx.doi.org/10.1590/S0034-89102011005000083

15. Machado MMT, Lima ÂSS, Bezerra Filho JG, Machado MFAS, Lindsay AC, Magalhães FB, et al. Characteristics of consultation and mothers' satisfaction in primary care for children under 5 years of age, Fortaleza, Brazil. Ciênc Saúde Coletiva. 2012;17(11):3125-33. http://dx.doi. org/10.1590/S1413-81232012001100028

16. Silva Júnior EB, Nunes LMN. Avaliação da Assistência Farmacêutica na atenção primária no município de Petrolina (PE). Arq Bras Ciênc Saúde. 2012;37(2):65-9. http://dx.doi.org/10.7322/abcs.v37i2.34

17. Costa JM, Pereira ML. Implantação da Atenção Farmacêutica em uma Unidade de Atenção Primária à Saúde do Brasil: avaliação qualitativa por uma equipe multiprofissional. Revista APS. 2012 [acesso em 2013 Aug 03];15(3). Disponível em: http://www.aps.ufff.br/index.php/aps/article/ view/1673

18. Cardoso MO, Vieira-da-Silva LM. Evaluation of primary care coverage in Salvador, Bahia State, Brazil (2000 to 2007). Cad Saúde Pública. 2012;28(7):1273-84. http://dx.doi.org/10.1590/S0102-311X2012000700006

19. Uchoa AC, Souza EL, Spinelli AFS, Medeiros RG, Peixoto DCS, Silva RAR, et al. Evaluation of user's satisfaction with the Family Health Program in the rural area of two smalls cities in Rio Grande do Norte State, Brazil. Physis: Rev Saúde Coletiva. 2011;21(3):1061-76. http://dx.doi. org/10.1590/S0103-73312011000300016

20. Rabetti AC, Freitas SFT. Evaluation of actions concerning systemic arterial hypertension in primary healthcare. Rev Saúde Pública. 2011;45(2):258-68. http://dx.doi.org/10.1590/S0034-89102011005000007

21. Pereira MJB, Abrahão-Curvo P, Fortuna CM, Coutinho SS, Queluz MC, Campos LVO, et al. Evaluation of organizational and performance features in a Basic Health Unit. Rev Gaúcha Enfermagem. 2011;32(1):48-55. http://dx.doi.org/10.1590/S1983-14472011000100006

22. Mendoza-Sassi RA, Cesar JA, Teixeira TP, Ravache C, Araújo GD, Silva TC. [Differences in prenatal care between health services under the Family Health Strategy and traditional primary care clinics in Rio Grande, Rio Grande do Sul State, Brazil]. Cad Saúde Pública. 2011;27(4):787-96. http:// dx.doi.org/10.1590/S0102-311X2011000400018

23. Leão CDA, Caldeira AP, Oliveira MMC de. Aspects of primary care for children: an evaluation of care-givers. Rev Bras Saúde Materno Infantil. 2011;11(3):323-34. http://dx.doi.org/10.1590/S1519-38292011000300013

24. Lafaiete RS, Motta MCS, Villa TCS. User satisfaction in the tuberculosis control program in a city in Rio de Janeiro, Brazil. Rev Lat Am Enfermagem. 2011;19(3):508-14. http://dx.doi.org/10.1590/S0104-11692011000300009

25. Gomes KO, Cotta RMM, Araújo RMA, Cherchiglia ML, Martins TCP. Primary health care - the "apple of the eye" of SUS: about social representations of the protagonists of the Unified Health System. Ciênc Saúde Coletiva. 2011;16:881-92. http://dx.doi.org/10.1590/S141381232011000700020

26. Costa JMBS, Silva MRF, Carvalho EF. The implementation analysis of the arterial hypertension care by the Family Health teams in Recife city (Pernambuco, Brazil). Ciênc Saúde Coletiva. 2011;16(2):623-33. http://dx.doi.org/10.1590/S1413-81232011000200026

27. Carmo TA, Andrade SM, Cerci Neto A. [Evaluation of an asthma control program in family health units]. Cad Saúde Pública. 2011;27(1):162-72. http://dx.doi.org/10.1590/S0102-311X2011000100017

28. Busato IMS, Gabardo MCL, França BHS, Moysés SJ, Moysés ST. Evaluation of the perception of the oral health teams of the municipal health department of Curitiba, Paraná State, regarding atraumatic restorative treatment (ART). Ciênc Saúde Coletiva. 2011;16:1017-22. http://dx.doi. org/10.1590/S1413-81232011000700034

29. Arakawa T, Arcêncio RA, Scatolin BE, Scatena LM, Ruffino-Netto A, Villa TCS. Accessibility to tuberculosis treatment: assessment of health service performance. Rev Lat Am Enfermagem. 2011;19(4):994-1002. http://dx.doi.org/10.1590/S0104-11692011000400019

30. Vieira-da-Silva LM, Esperidião MA, Viana SV, Alves VS, Lemos DVS, Caputo MC, et al. Avaliação da implantação de programa voltado para melhoria da acessibilidade e humanização do acolhimento aos usuários na rede básica. Salvador, 2005-2008. Rev Bras Saúde Mat Infant. 2010;10:s131-s143. http://dx.doi.org/10.1590/S1519-38292010000500012

31. Serra CG, Rodrigues PHA. Evaluation of reference and counter-reference in the Family Health Program at the Metropolitan Region of Rio de Janeiro (RJ, Brazil). Ciênc Saúde Coletiva. 2010;15:3579-86. http://dx.doi.org/10.1590/S1413-81232010000900033

32. Mendonça MHM, Martins MIC, Giovanella L, Escorel S. Challenges for human resources management from successful experiences of Family Health Strategy expansion. Ciênc Saúde Coletiva. 2010;15(5):2355-65. http://dx.doi.org/10.1590/S1413-81232010000500011

33. Lima MAA, Oliveira MAA, Ferreira HS. Reliability of anthropometric data obtained in children seen at the Primary Public Healthcare Service Network in Alagoas, Brazil. Rev Bras Epidemiol. 2010;13(1):69-82. http://dx.doi.org/10.1590/S1415-790X2010000100007 
34. Lana FCF, Lima AS. Prevention evaluation of HIV vertical transmission in Belo Horizonte, MG, Brazil. Rev Bras Enfermagem. 2010;63(4):587-94. http://dx.doi.org/10.1590/S0034-71672010000400014

35. Hoepfner C, Franco SC. Therapeutic inertia and control of high blood pressure in primary health care units. Arq Bras Cardiol. 2010;95(2):223-8. http://dx.doi.org/10.1590/S0066-782X2010005000095

36. Fischer TK, Peres KG, Kupek E, Peres MA. Primary dental care indicators: association with socioeconomic status, dental care, water fluoridation and Family Health Program in Southern Brazil. Rev Bras Epidemiol. 2010;13(1):126-38. http://dx.doi.org/10.1590/S1415-790X2010000100012

37. Cunha ABO, Vieira-da-Silva LM. Health services accessibility in a city of Northeast Brazil. Cad Saúde Pública. 2010;26(4):725-37. http://dx.doi. org/10.1590/S0102-311X2010000400015

38. Amaral AS, Tamaki EM, Sales CM, Renovato RD. Evaluation of the decentralization of the tuberculosis control program from secondary to primary level in the health system of Dourados-MS. Saúde Sociedade. 2010;19(4):794-802. http://dx.doi.org/10.1590/S0104-12902010000400007

39. Tavares M de FL, Mendonça MHM de, Rocha RM da. Health practices in the reorientation of primary care in Rio de Janeiro State, Brazil, from the perspective of users and health professionals. Cad Saúde Pública. 2009;25(5):1054-62. http://dx.doi.org/10.1590/S0102-311X2009000500012

40. Scatena LM, Villa TCS, Netto AR, Kritski AL, Figueiredo TMRM de, Vendramini SHF, et al. Difficulties in the accessibility to health services for tuberculosis diagnoses in Brazilian municipalities. Rev Saúde Pública. 2009;43(3):389-97. http://dx.doi.org/10.1590/S0034-89102009005000022

41. Pereira CRS, Patrício AAR, Araújo FAC, Lucena EES, Lima KC, Roncalli AG. Inclusion of oral health teams in the Family Health Program and its impact on the use of dental services. Cad Saúde Pública. 2009;25(5):985-96. http://dx.doi.org/10.1590/S0102-311X2009000500005

42. Nascimento AC, Moysés ST, Bisinelli JC, Moysés SJ. Oral health in the family health strategy: a change of practices or semantics diversionism. Rev Saúde Pública. 2009;43(3):455-62. http://dx.doi.org/10.1590/S0034-89102009000300009

43. Nagahama EEl. Avaliação da implantação de serviços de saúde reprodutiva no Município de Maringá, Paraná, Brasil. Cad Saúde Pública. 2009;25:s279-s290. http://dx.doi.org/10.1590/S0102-311X2009001400010

44. Motta MCS, Villa TCS, Golub J, Kritski AL, Ruffino-Netto A, Silva DF, et al. Access to tuberculosis diagnosis in Itaboraí City, Rio de Janeiro, Brazil: the patient's point of view. Int J Tuberc Lung Dis. 2009;13(9):1137-41.

45. Medina MG, Hartz ZMA. The role of the Family Health Program in the organization of primary care in municipal health systems. Cad Saúde Pública. 2009;25(5):1153-67. http://dx.doi.org/10.1590/S0102-311X2009000500022

46. Gouveia GC, Souza WV, Luna CF, Souza-Júnior PRB, Szwarcwald CL. User satisfaction in the Brazilian health system: associated factors and regional differences. Rev Bras Epidemiol. 2009;12(3):281-96. http://dx.doi.org/10.1590/S1415-790X2009000300001

47. Gomes KO, Cotta RMM, Euclydes MP, Targueta CL, Priore SE, Franceschini SCC. Evaluation of the impact of the Family Health Program in the epidemiology profile of the rural population of Airões, city of Paula Cândido (MG), 1992-2003. Ciênc Saúde Coletiva. 2009;14:1473-82. http:// dx.doi.org/10.1590/S1413-81232009000800020

48. Finkelsztejn A, Acosta LMW, Cristovam RA, Moraes GS, Kreuz M, Sordi AO, et al. Referrals from primary care to neurological assessment in the city of Porto Alegre, Brazil. Physis: Rev Saúde Coletiva. 2009;19(3):731-41. http://dx.doi.org/10.1590/S0103-73312009000300010

49. Figueiredo TMRM, Villa TCS, Scatena LM, Cardozo Gonzales RI, Ruffino-Netto A, Nogueira JA, et al. Performance of primary healthcare services in tuberculosis control. Rev Saúde Pública. 2009;43(5):825-31. http://dx.doi.org/10.1590/S0034-89102009005000054

50. Fernandes VBL, Caldeira AP, Faria AA de, Rodrigues Neto JF. Hospitalizations sensitive to primary care as an evaluation indicator for the Family Health Strategy. Rev Saúde Pública. 2009;43(6):928-36. http://dx.doi.org/10.1590/S0034-89102009005000080

51. Almeida CAL de, Tanaka OY. Perspectiva das mulheres na avaliação do Programa de Humanização do Pré-Natal e Nascimento. Rev Saúde Pública. 2009;43(1):98-104. http://dx.doi.org/10.1590/S0034-89102009000100013

52. Silva LMV, Formigli VLA. Health evaluation: problems and perspctives. Cad Saúde Pública. 1994;10(1):80-91. http://dx.doi.org/10.1590/S0102$311 \times 1994000100009$

53. Donabedian A. The quality of care. How can it be assessed? JAMA. 1988 Sep 23;260(12):1743-8. http://dx.doi.org/10.1001/ jama.1988.03410120089033

54. Worhern BR, Sanders JR, Fitzpatrick JL. Avaliação de programas. São Paulo: Gente; 2004.

55. Serapioni M. Métodos qualitativos e quantitativos na pesquisa social em saúde: algumas estratégias para a integração. Ciênc Saúde Coletiva. 2000;5(1):187-92. http://dx.doi.org/10.1590/S1413-81232000000100016

56. Bosi MLM, Mercado-Martinez FJ. Notas para um debate. In: Mercado-Martinez FJ, Bosi MLM, editors. Pesquisa qualitativa de serviços de saúde. Petrópolis: Vozes; 2004. p. 23-71.

57. Santos-Filho SB. Perspectives of the evaluation of Brazil's National Health Humanization Policy: conceptual and methodological aspects. Ciênc Saúde Coletiva. 2007;12(4):999-1010. http://dx. doi.org/10.1590/S1413-81232007000400021

58. Vitoria AM, Harzheim E, Takeda SP, Hauser L. Avaliação dos atributos da atenção primária à saúde em Chapecó, Brasil. Rev Bras Med Fam Comunidade. 2013 Oct 7;8(29):285-93. http://dx.doi.org/10.5712/rbmfc8(29)832

59. Hartz ZMA. Meta-evaluation of health management: challenges for "new public health". Ciênc Saúde Coletiva. 2012;17(4):832-4. http://dx.doi. org/10.1590/S1413-81232012000400004 